\title{
First-Principles Theory of Surface Thermodynamics and Kinetics
}

\author{
C. Stampfl, ${ }^{1}$ H. J. Kreuzer, ${ }^{2}$ S. H. Payne, ${ }^{2}$ H. Pfnür, ${ }^{3}$ and M. Scheffler ${ }^{1}$ \\ ${ }^{1}$ Fritz-Haber-Institut der Max-Planck-Gesellschaft, Faradayweg 4-6, D-14195 Berlin, Germany \\ ${ }^{2}$ Department of Physics, Dalhousie University, Halifax, Nova Scotia, Canada B3H $3 J 5$ \\ ${ }^{3}$ Institut für Festkörperphysik, Universität Hannover, Appelstrasse 2, \\ D-30176 Hannover, Germany
}

(Received 3 May 1999)

\begin{abstract}
Understanding the complex behavior of particles at surfaces requires detailed knowledge of both macroscopic and microscopic processes that take place; also certain phenomena depend critically on temperature and gas pressure. To link these processes we combine state-of-the-art microscopic, and macroscopic phenomenological, theories. We apply our theory to the $\mathrm{O} / \mathrm{Ru}(0001)$ system and calculate thermal desorption spectra, heat of adsorption, and the surface phase diagram. The agreement with experiment provides validity for our approach which thus identifies the way for a predictive simulation of surface thermodynamics and kinetics.
\end{abstract}

PACS numbers: 68.45.Da, 82.65.Dp, 82.65.My

The study of the physical and chemical processes that take place at gas-surface interfaces have long been an area of intense research. This interest is both fundamental as well as driven by the possible discovery of important technological applications, e.g., in the field of heterogeneous catalysis, corrosion, etc. [1,2]. With respect to the field of the theory of adsorption of gases on solid surfaces, advancement in recent years has developed in two distinct, albeit complimentary, directions: (i) electronic structure calculations, at best done by density-functional theory (DFT), to determine the geometries, energetics, and vibrational properties of adsorbate covered surfaces, and (ii) phenomenological models, both for the thermodynamics and the kinetics [3] of the adsorbate. If one can assume that the geometry of the solid surface does not change dramatically and that adsorption occurs at well defined sites, one frequently employs a lattice gas model. A number of parameters enter this type of model, such as the binding energies and vibrational frequencies of a single adparticle in the various adsorption sites, and their mutual lateral interactions with adparticles in close-by sites. Traditionally, these parameters are adjusted in the theory in order to fit a variety of experimental data such as phase diagrams, heats of adsorption, infrared spectra, and thermal desorption data, etc. Such an approach, while useful, is clearly not necessarily predictive in nature, nor the parameters unique, and may not capture the physics of the microscopic processes that are behind the "best-fit" adjusted "effective" parameters.
In this Letter, with the aim to improve upon this approach, we combine state-of-the-art procedures of (i) microscopic theories, i.e., DFT electronic structure calculations and (ii) macroscopic phenomenological approaches, i.e., lattice gas and rate equations, and Monte Carlo schemes. On doing this, we present a consistent first-principles-based approach for calculation of the thermodynamic and kinetic properties of an adsorbate, such as heats of adsorption, temperature programmed desorption (TPD) spectra, and the surface phase diagram. We have chosen the system of oxygen at $\mathrm{Ru}(0001)$ for which detailed structural [4-9], thermodynamic [10], and kinetic data $[11,12]$ exist. We will show that, with the present approach, a realistic description of these physical properties is indeed feasible.

The electronic structure calculations [13] are performed using DFT and the generalized gradient approximation (GGA) for the exchange-correlation functional [14] (hereafter denoted as DFT-GGA). We use the pseudopotential $[15,16]$ plane wave method and the supercell approach to model the surface. The positions of the $\mathrm{O}$ atoms and the top two $\mathrm{Ru}$ layers are fully relaxed. The DFT-GGA Ru pseudopotential yields a bulk hcp-fcc energy difference of $\approx-0.072 \mathrm{eV}$, in good agreement with all-electron calculations [17]. For further technical details of the calculations we refer to Refs. $[6,18]$.

To set up a lattice gas model for $\mathrm{O}$ on $\mathrm{Ru}(0001)$ we require a Hamiltonian, which we express as

$$
\begin{aligned}
H=E_{s}^{\mathrm{hcp}} \sum_{i} n_{i}+E_{s}^{\mathrm{fcc}} \sum_{i} n_{i}+\frac{1}{2}( & V_{1 n}^{\mathrm{hcp}} \sum_{i, a} n_{i} n_{i+a}+V_{1 n}^{\mathrm{fcc}} \sum_{i, a} n_{i} n_{i+a}+V_{1 n}^{\mathrm{hcp}-\mathrm{fcc}} \sum_{i, a^{\prime}} n_{i} n_{i+a^{\prime}}+V_{2 n}^{\mathrm{hcp}} \sum_{i, b} n_{i} n_{i+b} \\
& +V_{2 n}^{\mathrm{fcc}} \sum_{i, b} n_{i} n_{i+b}+V_{2 n}^{\mathrm{hcp}-\mathrm{fcc}} \sum_{i, b^{\prime}} n_{i} n_{i+b^{\prime}}+V_{3 n}^{\mathrm{hcp}} \sum_{i, c} n_{i} n_{i+c}+V_{3 n}^{\mathrm{fcc}} \sum_{i, c} n_{i} n_{i+c} \\
& \left.+V_{3 n}^{\mathrm{hcp}-\mathrm{fcc}} \sum_{i, c^{\prime}} n_{i} n_{i+c^{\prime}}+V_{\mathrm{trio}}^{\mathrm{hcp}} \sum_{i, a, a^{\prime \prime}} n_{i} n_{i+a} n_{i+a^{\prime \prime}}+V_{\mathrm{trio}}^{\mathrm{fcc}} \sum_{i, a, a^{\prime \prime}} n_{i} n_{i+a} n_{i+a^{\prime \prime}}+\ldots\right) .
\end{aligned}
$$


The different unit cells of the substrate surface are labeled by an index $i$ with $i+a$, etc., labeling neighboring cells, and we introduce occupation numbers $n_{i}=0$ or 1 , depending on whether a site in cell $i$ is empty or occupied. Overcounting of cells is excluded in the summations. Equation (1) includes consideration of hcp and fcc sites. The indices $a^{\prime}, b^{\prime}$, and $c^{\prime}$ indicate that the first, second, and third neighbor distances between atoms in hcp and fcc sites are different than when they occupy the same type of sites. Here $E_{s}^{\mathrm{hcp}}=\left|V_{0}\right|-k_{B} T \ell n q_{3}$ is the binding energy of an isolated particle in an hcp site. $\left|V_{0}\right|$ is the depth of the adsorption potential with reference to the energy of a gas phase molecule which adsorbs dissociatively. $q_{3}$ is the partition function of the atom accounting for its vibration perpendicular, and its frustrated translation parallel, to the surface. $V_{1 n}^{\mathrm{hcp}}, V_{2 n}^{\mathrm{hcp}}$ and $V_{3 n}^{\mathrm{hcp}}$ are the first, second, and third neighbor interaction energies between two adsorbed $\mathrm{O}$ atoms in hep sites. The analogous terms labeled "fcc" represent the same quantities but for adsorption in fcc sites. Terms labeled $V^{\text {hcp-fcc }}$ represent the interactions between atoms in hcp and fcc sites. Trio (and higher cluster) interaction energies, $V_{\text {trio }}$, account for additional modifications because the interaction between two adsorbed $\mathrm{O}$ atoms is changed, when a third adatom is close by. In fact, depending on the angles and distances, there are three different trio interactions taken into account.

We determine the lateral interaction energies required in Eq. (1) from DFT-GGA calculations of ordered structures of $\mathrm{O}$ on $\mathrm{Ru}(0001)$ (see Fig. 1). The adsorption energy of a single oxygen atom on $\mathrm{Ru}(0001)$ is obtained using a $(3 \times 3)$ structure with coverage $\theta=1 / 9$. With this large O-O separation $(8.26 \AA)$, which corresponds to the fifth nearest-neighbor distance of like sites, lateral interactions are negligible. The adsorption energy, with respect to $1 / 2 \mathrm{O}_{2}$, is then expressed as

$$
E_{a}^{\theta=1 / 9}=E_{\text {total }}^{\mathrm{O} / \mathrm{Ru}}-E_{\text {total }}^{\mathrm{Ru}}-\frac{1}{2} E_{\text {total }}^{\mathrm{O}_{2}} .
$$

Here $E_{\text {total }}^{\mathrm{O} / \mathrm{Ru}}, E_{\text {total }}^{\mathrm{Ru}}$, and $E_{\text {total }}^{\mathrm{O}_{2}}$ are the total energies of the $(3 \times 3)-\mathrm{O} / \mathrm{Ru}(0001)$ system, the clean $\mathrm{Ru}$ surface, and a free $\mathrm{O}_{2}$ molecule, respectively. Expressions analogous to Eq. (2) have been used to derive the adsorption energies for the other coverages (see Table I). We expand the adsorption energies in terms of two- and three-body interactions. The interaction parameters are derived from the equations:

$$
\begin{aligned}
E_{a}^{\theta=2 / 9}= & V_{0}+\frac{1}{2}\left(V_{1 n}+V_{3 n}\right), \\
E_{a}^{\theta=1 / 4}= & V_{0}+3 V_{3 n}, \\
E_{a}^{\theta=1 / 3}= & V_{0}+3 V_{2 n}, \\
E_{a}^{\theta=1 / 2}= & V_{0}+V_{1 n}+V_{2 n}+3 V_{3 n}+V_{\mathrm{lt}}, \\
E_{a}^{\theta=2 / 3}= & V_{0}+\frac{3}{2} V_{1 n}+3 V_{2 n}+\frac{3}{2} V_{3 n}+3 V_{\mathrm{bt}}, \\
E_{a}^{\theta=3 / 4}= & V_{0}+2 V_{1 n}+2 V_{2 n}+3 V_{3 n}+2 V_{\mathrm{lt}}+2 V_{\mathrm{bt}} \\
& +\frac{2}{3} V_{\mathrm{tt}}, \\
E_{a}^{\theta=1.0}= & V_{0}+3\left(V_{1 n}+V_{2 n}+V_{3 n}\right)+3 V_{\mathrm{lt}}+6 V_{\mathrm{bt}} \\
& +2 V_{\mathrm{tt}},
\end{aligned}
$$

where $V_{\mathrm{lt}}, V_{\mathrm{bt}}$, and $V_{\mathrm{tt}}$ are linear, bent, and triangular trios, respectively (indicated in Fig. 1). We point out that only in the $(3 \times 3)-2 \mathrm{O}$ structure at $\theta=2 / 9$ can the atoms at nearest-neighbor sites move significantly from the locally threefold symmetric adsorption sites to reduce the repulsion, $V_{1 n}$. However, with strong-nearest neighbor repulsion, isolated nearest-neighbor pairs are highly improbable at any coverage. We have therefore calculated $E_{a}^{\theta=2 / 9}$ for atoms at locally threefold symmetric sites because if we were to use the relaxed structure we would need to include higher many-body interactions (e.g., longer-ranged trios, quartos, and quintos, etc.) to account for the movement of the atoms back to the ideal threefold sites which occurs for higher coverages.

We thus have, for $\mathrm{O}$ atoms in hcp sites (and analogously for $\mathrm{O}$ atoms in fcc sites), seven equations for six unknowns. Using the first six of these equations, we obtain the interaction energies listed in Table II. The accuracy of these interaction values is gauged by calculating the adsorption energy of the monolayer (seventh equation); we find a discrepancy of $0.034 \mathrm{eV}$ smaller than that of the DFT-GGA value. Along with our derived values in Table II, we give in brackets the interaction energies determined by Piercy et al. [10] for their best fit to the experimental $\mathrm{O} / \mathrm{Ru}(0001)$ phase diagram. We also include our derived interaction parameters for structures involving $\mathrm{O}$ atoms in hcp and fcc sites (last three diagrams of Fig. 1) obtained by writing down the appropriate equations in an analogous manner to those listed above.

To complete the specification of our Hamiltonian, we need the vibrational frequencies of an $\mathrm{O}$ atom relative to the $\mathrm{Ru}$ surface. These frequencies can also be calculated using density-functional theory, for example, the vibration of oxygen normal to the surface at the $\bar{\Gamma}$ point (calculated to be $\nu_{z} \approx 509 \mathrm{~cm}^{-1}$ ).
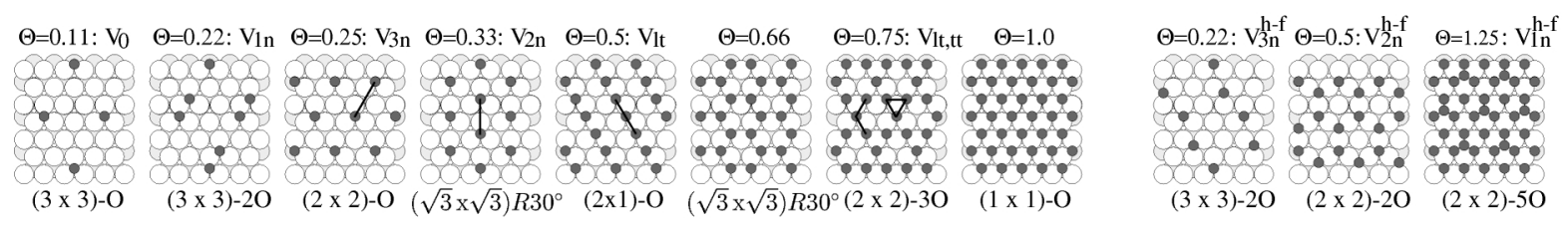

FIG. 1. Adsorbate structures calculated using DFT-GGA. (For the first eight diagrams, analogous calculations were also performed for $\mathrm{O}$ in fcc sites.) Small circles represent $\mathrm{O}$ atoms and large circles represent $\mathrm{Ru}$ atoms. 
TABLE I. Adsorption energies (in $\mathrm{eV}$ ) for $\mathrm{O}$ on $\mathrm{Ru}(0001)$ with respect to $1 / 2 \mathrm{O}_{2}$ for various coverages.

\begin{tabular}{|c|c|c|c|c|c|c|c|c|c|c|c|c|}
\hline Site & $V_{0}=E_{a}^{\theta=1 / 9}$ & $E_{a}^{\theta=2 / 9}$ & $E_{a}^{\theta=1 / 4}$ & $E_{a}^{\theta=1 / 3}$ & $E_{a}^{\theta=1 / 2}$ & $E_{a}^{\theta=2 / 3}$ & $E_{a}^{\theta=3 / 4}$ & $E_{a}^{\theta=1}$ & Site & $\begin{array}{c}E_{a, \mathrm{hcp}-\mathrm{fcc}}^{\theta=2 / 9} \\
\end{array}$ & $\begin{array}{c}E_{a, \mathrm{hcp}-\mathrm{fcc}}^{\theta=1 / 2} \\
\end{array}$ & $\begin{array}{l}E_{a, \mathrm{hcp}-\mathrm{fcc}}^{\theta=5 / 4} \\
\end{array}$ \\
\hline hcp & -2.503 & -2.417 & -2.577 & -2.370 & -2.307 & -2.150 & -2.0 & -1.895 & & -2.294 & -2.209 & -1.492 \\
\hline fcc & -2.152 & -2.107 & -2.145 & -2.105 & -2.025 & -2.015 & -1.942 & -1.865 & & & & \\
\hline
\end{tabular}

We now proceed to calculate the temperature programmed thermal desorption spectra. Writing the kinetic equation for adsorption and desorption as $d \theta / d t=$ $R_{\text {ad }}-R_{\text {des }}$, we obtain, for an atomic adsorbate in contact with a gas of diatomic homonuclear molecules, the rate of adsorption $R_{\mathrm{ad}}=2 S_{\mathrm{dis}}(\theta, T) P_{m} a_{s} \lambda_{m} / h$. Here $P_{m}$ is the molecular pressure above the surface, $a_{s}$ is the area of one unit cell of the substrate surface, $\lambda_{m}=h /\left(2 \pi m k_{B} T\right)^{1 / 2}$ is the thermal wavelength of a molecule of mass $m$, and $S_{\text {dis }}(\theta, T)$ is the dissociative sticking coefficient. For the rate of desorption we have [3]

$$
\begin{aligned}
R_{\mathrm{des}}= & 2 S_{\mathrm{dis}}(\theta, T) a_{s} \frac{k_{B} T}{h \lambda_{m}^{2}} \frac{Z_{v r}}{q_{3}^{2}} \frac{\theta^{2}}{(1-\theta)^{2}} \\
& \times e^{-2\left|V_{0}\right| / k_{B} T} e^{2 \mu^{(\text {lat })} / k_{B} T} .
\end{aligned}
$$

Here $Z_{v r}$ is the partition function accounting for the internal vibrations and rotations of $\mathrm{O}_{2}$ in the gas phase. $\mu^{\text {(lat) }}$ is the contribution to the chemical potential of the adsorbate due to the lateral interactions in the Hamiltonian [Eq. (1)] and is calculated here using transfer matrix techniques. Regarding the sticking coefficient, we note that dissociation is not activated initially, but at (local) coverages of $\theta \geqslant 0.5$, it is hindered by an energy barrier $[6,12]$. Under these circumstances, to obtain the coverage and temperature dependence of the sticking coefficient $a b$ initio would be a significant undertaking. Therefore we use an analytic expression which well approximates the measured behavior in the temperature regime of desorption [12]: The sticking coefficient drops approximately as $(2 / 3-\theta)^{2}$ and for coverages above $2 / 3$ it remains very small up to a monolayer. The actual equation we use is $S_{\text {dis }}(\theta)=S_{0} \exp \left[-(\theta / \sigma)^{2}\right]$, with $S_{0}=0.27$ and $\sigma=0.3$.

Our calculated TPD spectra [19] are shown in Fig. 2 which are compared to recent experimental data [20]. For low initial coverage we note that in the theory the oxygen desorbs at about $100 \mathrm{~K}$ higher temperature than in experiment, reflecting an overbinding of the $\mathrm{O}$ atoms. We believe that this size error is typical for present day

TABLE II. DFT-GGA calculated interaction energies (in eV) for $\mathrm{O}$ on $\mathrm{Ru}(0001)$. In parenthesis we list the interaction parameters used by Piercy et al. [10].

\begin{tabular}{ccccccc}
\hline \hline Site & $V_{1 n}$ & $V_{2 n}$ & $V_{3 n}$ & $V_{1 \mathrm{t}}$ & $V_{\mathrm{bt}}$ & $V_{\mathrm{tt}}$ \\
\hline hcp & 0.265 & 0.044 & -0.025 & -0.039 & -0.046 & 0.058 \\
& $(0.23)$ & $(0.069)$ & $(-0.023)$ & & & \\
fcc & 0.158 & 0.016 & 0.002 & -0.052 & -0.044 & 0.076 \\
& $(0.069)$ & & & & & \\
fcc-hcp & 0.586 & 0.101 & 0.033 & & & \\
\hline \hline
\end{tabular}

state-of-the-art calculations and don't see that (or how) better accuracy will be achieved in the coming five to ten years. Aside from this, it can be seen that the theoretical spectra exhibit all of the features of the experimental data: (i) a shift of the peak maxima to lower temperatures for higher initial coverages (appropriate for second order desorption and/or repulsive interactions) and (ii) a steepening of the leading edge for higher initial coverages. In our theory this steepening is a reflection of two facts: Firstly, for increasing coverages, the repulsive next-nearest-neighbor interactions lower the binding energy and thus lower the onset of desorption, broadening the TPD spectra and steepening the rising edge; secondly, and more importantly, is the effect of the rapidly decreasing sticking probability for increasing coverage. Because the sticking coefficient is much smaller for coverages above $2 / 3 \mathrm{ML}$, desorption is delayed to higher temperatures and the last third of a monolayer desorbs over a very narrow temperature range. We can trace the two shoulders (at 1100 and $1300 \mathrm{~K}$ ) to the synergy of the interactions which at lower temperatures lead to the formation of the $(2 \times 1)$ and $(2 \times 2)$ ordered structures, which will also be seen in the heat of adsorption.

We investigated the affect of spillover into fcc sites; we found that the overall features of the TPD spectra remain essentially unchanged whether spillover is included or not. We have also tested the importance of the trio interactions on desorption. Neglecting them increases the overall repulsion for coverages larger than 2/3 ML and consequently broadens the TPD spectra, reducing the agreement with experiment. We therefore conclude that for high $\mathrm{O}$-coverages, trio interactions play an important role.
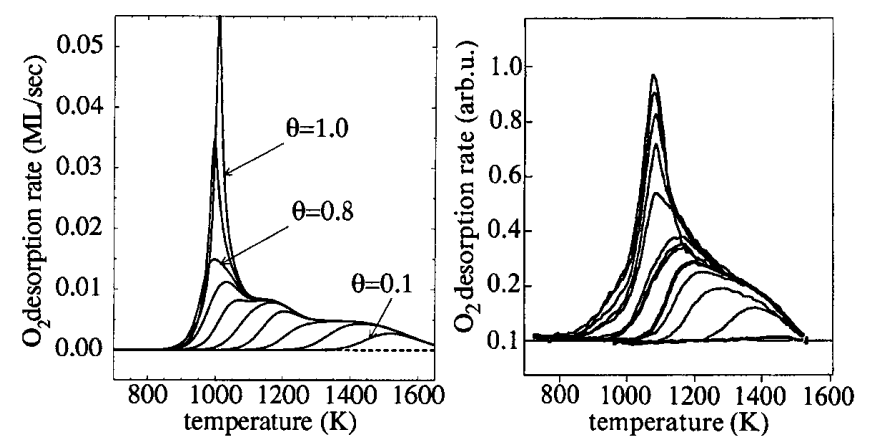

FIG. 2. Theoretical (left panel) and experimental [20] (right panel) TPD spectra (heating rate of $6 \mathrm{~K} / \mathrm{s}$ ). For the theoretical results, initial coverages are $\Theta=0.1$ to 1.0 in steps of 0.1 ; the experimental results also span the initial coverage region of $\theta \rightarrow 0$ to $1 \mathrm{ML}$. 


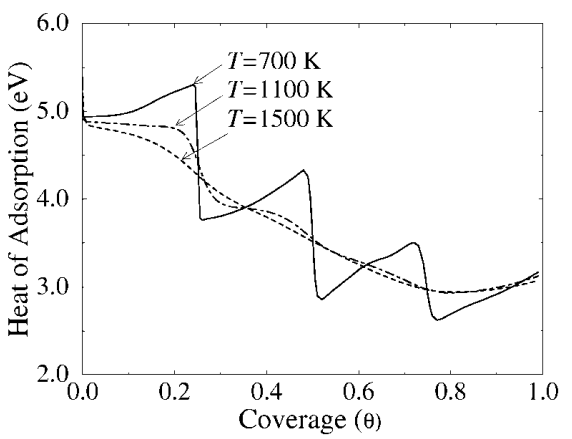

FIG. 3. The heat of adsorption as a function of coverage for temperatures of $T=700$ (sharpest features), 1100 , and $1500 \mathrm{~K}$.

Having the chemical potential as a function of temperature and coverage, we can also calculate the equilibrium properties of the adsorbate, such as adsorption isobars. As an example, we present in Fig. 3 the isosteric heat of adsorption [3] for a few temperatures. At the highest temperature, a smooth decrease is observed. At the lowest temperature, sharp peaks (and decreases) at 1/4, 1/2, 3/4, and $1 \mathrm{ML}$ occur. These coverages correspond to each of the ordered phases that form in nature, i.e., $(2 \times 2)$-O [4], $(2 \times 1)-\mathrm{O}$ [5], $(2 \times 2)-3 \mathrm{O}$ [7-9], and $(1 \times 1)-\mathrm{O}[6]$. [Note that the existence of the $(2 \times 2)-3 \mathrm{O}$ and $(1 \times 1)$-O adsorption phases was at first predicted by DFT-GGA calculations [18] and subsequently confirmed by experiment (a nice success of DFT).] The rises in between originate from the third neighbor attractions, and also from the trios for the higher coverages. Our results show no tendency for the stability of a $\theta=1 / 3$ phase, i.e., a $(\sqrt{3} \times \sqrt{3}) R 30^{\circ}$ structure, in agreement with experiment.

We now turn to the surface phase diagram. From Table II it can be seen that the overall agreement of the interaction parameters determined from our densityfunctional calculations and from the best fit to experiment in Ref. [10] is, in general, astonishingly good; but there are some significant differences (i.e., more than $50 \%$ for $\left.V_{1 n}^{\mathrm{fcc}}\right)$ which could be expected. We find that our Monte Carlo simulations yield a surface phase diagram rather similar to that of Piercy et al. [10], where coverage up to half a monolayer was considered. Our present simulations also included higher $\mathrm{O}$ coverages and correctly predict the formation of the $(2 \times 2)-3 \mathrm{O}$ phase.

In summary, we have presented a first-principles-based approach for calculation of the thermodynamics and kinetics of an adsorbate on a surface. We used densityfunctional theory to create a lattice gas Hamiltonian from which we evaluated the partition function. Our theoretical temperature programmed thermal desorption spectra, heats of adsorption, and the surface phase diagram for $\mathrm{O}$ on $\mathrm{Ru}(0001)$ show very good overall agreement with available experimental results, providing confidence in our approach. We found that trio interactions, as well as the sticking coefficient, play an important role in the TPD spectra of the present system. The attractive trio interactions also apparently help stabilize the higher coverage $(2 \times 1)$-O, $(2 \times 2)$-3O , and $(1 \times 1)$-O phases. The effect of spillover into fcc sites was found to have a minimal effect on the TPD spectra.

[1] The Chemical Physics of Solid Surfaces and Heterogeneous Catalysis, edited by D. A. King and D. P. Woodruff, Studies in Surface Science and Catalysis Vol. 4 (Elsevier, Amsterdam, 1982).

[2] Equilibria and Dynamics of Gas Adsorption on Heterogeneous Solid Surfaces, edited by W. Rudziński, W. A. Steele, and G. Zgrablich (Elsevier, Amsterdam, 1997), Vol. 104.

[3] H. J. Kreuzer and S. H. Payne, in Ref. [2].

[4] M. Lindroos, H. Pfnür, D. Held, and D. Menzel, Surf. Sci. 222, 451 (1989).

[5] H. Pfnür, D. Held, M. Lindroos, and D. Menzel, Surf. Sci. 220, 43 (1989).

[6] C. Stampfl, S. Schwegmann, H. Over, M. Scheffler, and G. Ertl, Phys. Rev. Lett. 77, 3371 (1996).

[7] K. L. Kostov, M. Gsell, P. Jakob, T. Moritz, W. Widdra, and D. Menzel, Surf. Sci. 394, L138 (1997).

[8] Y.D. Kim, S. Wendt, S. Schwegmann, H. Over, and G. Ertl, Surf. Sci. 418, 267 (1998).

[9] M. Gsell, M. Stichler, P. Jakob, and D. Menzel, Isr. J. Chem. 38, 339 (1999).

[10] P. Piercy, K. De'Bell, and H. Pfnür, Phys. Rev. B 45, 1869 (1992).

[11] T.E. Madey, H. A. Engelhardt, and D. Menzel, Surf. Sci. 48, 304 (1975).

[12] L. Surnev, G. Rangelov, and G. Bliznakov, Surf. Sci. 159, 299 (1985).

[13] R. Stumpf and M. Scheffler, Comput. Phys. Commun. 79, 447 (1994); M. Bockstedte, A. Kley, J. Neugebauer, and M. Scheffler, Comput. Phys. Commun. 107, 187 (1997).

[14] J. P. Perdew, J. A. Chevay, S. H. Vosko, K. A. Jackson, M. R. Pederson, D. J. Singh, and C. Fiolhais, Phys. Rev. B 46, 6671 (1992).

[15] N. Troullier and J. L. Martins, Phys. Rev. B 43, 1993 (1991).

[16] M. Fuchs and M. Scheffler, Comput. Phys. Commun. 119, 67 (1999).

[17] H. L. Skriver, Phys. Rev. B 31, 1909 (1985).

[18] C. Stampfl and M. Scheffler, Phys. Rev. B 54, 2868 (1996); Surf. Sci. 377-379, 808 (1997).

[19] ASTEK package written by H. J. Kreuzer and S. H. Payne (available from Helix Science Applications, Box 49, Site 3, R.R.5, Armdale, Nova Scotia, Canada B3L 455).

[20] A. Böttcher, H. Niehus, S. Schwegmann, H. Over, and G. Ertl, J. Phys. Chem. 101, 11185 (1997). 